\title{
Boundary states as holographic duals of trivial spacetimes
}

\author{
Masamichi Miyaji, ${ }^{a}$ Shinsei Ryu, ${ }^{b}$ Tadashi Takayanagi ${ }^{a, c}$ and Xueda Wen ${ }^{b}$ \\ ${ }^{a}$ Yukawa Institute for Theoretical Physics, \\ Kyoto University, Kyoto 606-8502, Japan \\ ${ }^{b}$ Department of Physics, University of Illinois at Urbana-Champaign, \\ 1110 West Green St, Urbana IL 61801, U.S.A. \\ ${ }^{c}$ Kavli Institute for the Physics and Mathematics of the Universe, \\ University of Tokyo, Kashiwa, Chiba 277-8582, Japan \\ E-mail: masamichi.miyaji@yukawa.kyoto-u.ac.jp, ryuu@illinois.edu, \\ takayana@yukawa.kyoto-u.ac.jp, xwen4@illinois.edu
}

ABSTRACT: We study real-space quantum entanglement included in conformally invariant boundary states in conformal field theories (CFTs). We argue that boundary states essentially have no real-space entanglement, except for constant contributions from long range topological entanglement, by computing the entanglement entropy when the system is bipartition into two spatial regions. From the viewpoint of classical gravity duals in holography, this shows that boundary states are dual to trivial spacetimes of zero spacetime volume. We also point out that a continuous multiscale entanglement renormalization ansatz (cMERA) for any CFTs can be formulated by employing a boundary state as its infrared unentangled state with an appropriate regularization. Exploiting this idea, we propose an approximation scheme of cMERA construction for general CFTs.

Keywords: AdS-CFT Correspondence, Boundary Quantum Field Theory, Holography and condensed matter physics (AdS/CMT), Renormalization Group

ARXIV EPRINT: 1412.6226 


\section{Contents}

1 Introduction 1

2 Boundary states as maximally entangled states 3

3 No real-space entanglement in boundary states 5

3.1 Boundary states from relevant perturbations 5

$\begin{array}{ll}3.2 & \text { Correlation functions }\end{array}$

3.3 A free field calculation of entanglement entropy of boundary states $\quad 7$

4 Boundary states as holographic duals of trivial spacetimes 9

4.1 AdS/CFT and holographic entanglement entropy 9

$\begin{array}{lll}4.2 & \text { An explicit construction in AdS/BCFT } & 10\end{array}$

5 cMERA for general CFTs and boundary states 11

$\begin{array}{lll}5.1 & \text { MERA and cMERA } & 12\end{array}$

$\begin{array}{ll}5.2 \text { IR states for general CFTs } & 15\end{array}$

$\begin{array}{lll}5.3 & \text { A general ansatz for cMERA } & 16\end{array}$

$\begin{array}{lll}\text { 5.3.1 Free massless scalar CFTs } & 16\end{array}$

$\begin{array}{ll}\text { 5.3.2 Free massless fermion CFT in two dimensions } & 17\end{array}$

$\begin{array}{lll}\text { 5.3.3 } & \text { cMERA for general 2d CFTs } & 18\end{array}$

6 Conclusions and discussion $\quad 18$

A A lattice model calculation of entanglement entropy of boundary states 20

B Example: cMERA for free scalar field theory 23

$\begin{array}{ll}\text { B.1 Construction of cMERA } & 23\end{array}$

B.2 Scale transformation in massless scalar 24

$\begin{array}{lll}\text { B.3 Massive scalar case } & 25\end{array}$

$\begin{array}{lll}\text { B.4 Circle compactification } & 26\end{array}$

\section{Introduction}

Recently, there have been important progresses in understanding the structures of spacetimes in gravitational theories. The holographic principle predicts that spacetimes in a gravitational theory are emergent from more fundamental degrees of freedom [1-3], as explicitly realized in the AdS/CFT correspondence [4]. Moreover, latest studies strongly suggest that these fundamental degrees of freedom are given by quantum entanglement in quantum many-body systems. The holographic formula of the entanglement entropy 
offers one manifestation of this connection between structures of spacetimes and quantum entanglement [5-11]. Furthermore, an explicit map between the structure of quantum entanglement and geometries of gravitational theories may be established by using the tensor network construction of many-body quantum state, in particular MERA (multi-scale entanglement renormalization ansatz) [12-14], as noted in [15]. A continuum version of MERA, called cMERA is available [16] and its connection to the AdS/CFT correspondence has been studied in [17]. Another interesting network construction was argued to be dual to gravity in [18]. Refer to, e.g., [19-26] for more progresses in this subject.

In this paper, we consider a holographic counterpart of the most fundamental spacetime: a trivial spacetime with vanishing spacetime volume, or in other words just a pointlike universe. As the holographic entanglement entropy shows, the area of spacetime is proportional to the entanglement entropy. Therefore, a trivial spacetime corresponds to a quantum state with no real-space entanglement, at least in the classical gravity limit. Once quantum fluctutions are taken into account, a quantum state with no real-space entanglement would be dual to spacetime of planck size volume. Indeed, the area law formula of holographic entanglement entropy [5-9] and its generalization to higher derivative gravities [27-30] can only be applied to spacetimes in classical gravity, which are much larger than the planck size.

From the viewpoint of its dual conformal field theory (CFT) in the setup of AdS/CFT, such a quantum state with no entanglement is rather non-trivial, since for typical states in CFTs, such as the CFT vacuum states, the real-space entanglement entropy scales with the size of the subregion of our interest and thus is far from vanishing [5-9, 31,32]. One of the main purposes of this paper is to point out that such an unentangled quantum state can be obtained as the conformally invariant boundary states in CFTs. The boundary states play an important role in CFTs because they provide general descriptions of boundary conformal field theories (BCFTs). In 2d CFT, the Cardy states [33] involve maximally entangled states of the holomorphic and antiholomorphic sectors of the CFTs, called the Ishibashi states. While this entanglement between holomorphic and antiholomorphi sectors has been commonly discussed, in this paper, we discuss real space entanglement included in boundary states by bipartitioning the total system into two spatial regions.

Identification of boundary states as a holographic dual to empty spacetimes also helps us to find a construction of cMERA for generic CFTs. In cMERA, target states (e.g., ground states) are constructed from a reference state (called IR state) without any real space entanglement by adding quantum entanglement. Based on the above considerations, we claim that for generic CFTs boundary states can be taken as IR states in cMERA. The IR state corresponds to a trivial spacetime. Starting from such an empty spacetime, emergent spacetimes can be constructed by adding quantum entanglement. Identification of the IR states is an important technical step toward the cMERA construction of the ground states of generic CFT. By using the new cMERA ansatz using a CFT boundary state as its IR state, we will show that we can improve the previous result for the $(1+1) \mathrm{d}$ free fermion in [16]. The cMERA state in the latter correctly reproduces the true ground state only at low energies, while the cMERA state in our new construction is valid for arbitrary energy scale. Furthermore, using boundary states as IR states, we propose an 
ansatz of cMERA type for ground states of general 2d CFTs. Using the Cardy formula, we can then derive the information metric of the ansatz [17], which is consistent with the previous free field calculations, and with the holographic interpretation.

This paper is organized as follows: in section 2, we give a quick overview of the construction of boundary states in CFTs and interpret them as the maximally entangled states. In section 3, we show that the boundary states have no real-space entanglement except for possible topological entanglement. In section 4, we argue that boundary states are holographically dual to trivial spacetimes. In section 5, we will start with a review of (c)MERA. Later we will argue that a general construction of cMERA for CFTs can be obtained by using the boundary states. In section 6 , we summarize our conclusions and discuss future problems. In the appendix A, we present a lattice model calculation of entanglement entropy of boundary states. In the appendix B, we show some useful details of cMERA for a free scalar field theory.

\section{Boundary states as maximally entangled states}

In this section, we will review the definition of boundary states in CFTs, mainly on twodimensional (2d) CFTs for illustrative purposes. We will then explain an interpretation of boundary states as maximally entangled states.

Consider a $2 \mathrm{~d}$ CFT defined on a spatial cylinder described by the coordinate $(t, x)$, where $t$ is the time coordinate and $x$ is the space coordinate with the periodicity $2 \pi$. We consider a quantum state at $t=0$ defined by the path-integral during the time period $-\epsilon<t<0$. At the time $t=-\epsilon$, we assume that there is a space-like boundary extending for all values of $x$. Finally we take the limit $\epsilon \rightarrow 0$. The state defined in this way is called the boundary state $|B\rangle$, and depends on the choice of the boundary conditions. Especially we are interested in the boundary conditions which preserve a half of original Virasoro symmetries - such theories are called boundary conformal field theories (BCFTs). More explicitly, boundary states in BCFTs are defined by the condition

$$
\left(L_{n}-\tilde{L}_{-n}\right)|B\rangle=0 .
$$

where $\left\{L_{n}\right\}_{n \in \mathbb{Z}}$ and $\left\{\tilde{L}_{n}\right\}_{n \in \mathbb{Z}}$ are two sets of Virasoro generators, for the left- and rightmoving sector, respectively. A simple solution to this condition is given by

$$
\left|I_{h}\right\rangle \equiv \sum_{\vec{k}}|\vec{k}, h\rangle_{L} \otimes|\vec{k}, h\rangle_{R}
$$

where $|\vec{k}, h\rangle_{L}$ is an orthonormal basis of the left-moving Verma module constructed from a

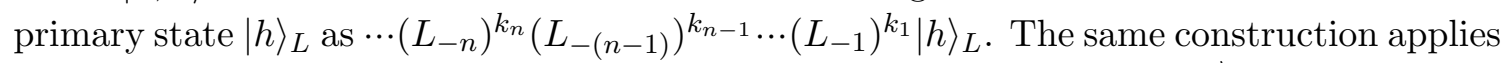
to the right-moving Virasoro algebra, and gives an orthonormal basis $|\vec{k}, h\rangle_{R}$. This state $\left|I_{h}\right\rangle$ is called the Ishibashi state for the primary state $|h\rangle$ [34]. The boundary states which correspond to physical boundary conditions are the so-called Cardy states [33], denoted by $\left|C_{\alpha}\right\rangle$. The Cardy states satisfy a consistency condition, so-called Cardy's condition or open-closed duality for partition functions on cylinders, which requires that the same results 
should be obtained both in open and closed string calculations. In general, the Cardy states (labeled by the index $\alpha$ ) are given by special linear combinations of Ishibashi states:

$$
\left|C_{\alpha}\right\rangle=\sum_{h} B_{\alpha, h}\left|I_{h}\right\rangle
$$

where $h$ runs over all primaries.

From the form (2.2), it is clear that the Ishibashi states are maximally entangled states of the left- and right-moving sectors of the CFT. The boundary states are singular in that their norms are infinite, and hence it is useful to introduce a regularization. A simple way to regularize the norms is to perform the Euclidean time evolution by $e^{-\epsilon H}$, where $H=L_{0}+\tilde{L}_{0}-\frac{c}{12}$. Then the Ishibashi states look like

$$
\frac{1}{\sqrt{Z(\epsilon)}} \sum_{\vec{k}} e^{-\epsilon E(\vec{k})}|\vec{k}, h\rangle_{L} \otimes|\vec{k}, h\rangle_{R},
$$

where $E(\vec{k})$ is the eigenvalue of $H$ and we defined $Z(\epsilon)=\sum_{\vec{k}} e^{-2 \epsilon E(\vec{k})}$. If we trace out the right-moving part we find that the reduced density matrix looks like

$$
\rho(h, \epsilon)=\frac{1}{Z(\epsilon)} \sum_{\vec{k}} e^{-2 \epsilon E(\vec{k})}|\vec{k}, h\rangle_{L L}\langle\vec{k}, h| .
$$

This is regarded as the thermal distribution at temperature $T=1 / 2 \epsilon$ of left-moving modes. Therefore the entanglement entropy between the left- and right-moving sectors coincides with the thermal entropy $S_{t h}(h, \epsilon)$ of the either sector. These facts were noted earlier in [26, $35]$ for free field theories. If we turn to the Cardy states with the same $\epsilon$ regularization, the reduced density matrix after tracing out the right-moving part is given by

$$
\frac{1}{\sum_{h}\left(B_{\alpha, h}\right)^{2}} \sum_{h}\left(B_{\alpha, h}\right)^{2} \rho(h, \epsilon)
$$

A few comments are in order. First, it is straightforward to generalize the above construction of the Ishibashi states to those in the presence of current algebras. While we omit details, it may be useful to remember that for the $\mathrm{U}(1)$ current algebra we can have a compact expression of the Ishibashi states:

$$
\left|I_{h( \pm)}\right\rangle=\exp \left( \pm \frac{1}{\sqrt{k}} \sum_{n=1}^{\infty} \frac{1}{n} J_{-n} \tilde{J}_{-n}\right)|h\rangle
$$

where the "level" $k$ is a positive integer, and $\left(J_{n}, \tilde{J}_{n}\right)$ are the generators of left- and rightmoving $\mathrm{U}(1)$ current algebras which satisfy $\left[J_{n}, J_{m}\right]=\left[\tilde{J}_{n}, \tilde{J}_{m}\right]=k n \delta_{n+m}$. This Ishibashi state satisfies the boundary conditions $\left(J_{n} \mp \tilde{J}_{-n}\right)|B\rangle=0$.

Second, it is straightforward to generalize the definition of boundary states in higherdimensional CFTs, though we have less analytical control compared with those in $2 \mathrm{~d}$ CFTs. For example, for a free massless scalar in $(d+1)$-dimensional flat spacetime, boundary states 
which correspond to the Neumann (+) and Dirichlet $(-)$ boundary conditions are explicitly given by

$$
|B( \pm)\rangle=\exp \left[ \pm \frac{1}{2} \int d^{d} k a_{\vec{k}}^{\dagger} a_{-\vec{k}}^{\dagger}\right]|0\rangle
$$

where $a_{\vec{k}}^{\dagger}$ is the creation operator of a momentum $\vec{k}$ scalar particle (see also the appendix $\mathrm{A}$ in this paper). This state again can be viewed as the maximally entangled state:

$$
|B( \pm)\rangle=\prod_{\vec{k}, k_{1}>0} \sum_{n_{\vec{k}}=0}^{\infty}( \pm 1)^{n_{\vec{k}}}\left|n_{\vec{k}}\right\rangle_{L} \otimes\left|n_{\vec{k}}\right\rangle_{R}
$$

where we decomposed the Hilbert space into those with $k_{1}>0$ (right-moving) and those with $k_{1}<0$ (left-moving), where $k_{1}$ is a component of the $d$-dimensional vector $\vec{k}$. We also define the number states $\left|n_{\vec{k}}\right\rangle_{L, R}$ as usual: $\left|n_{\vec{k}}\right\rangle_{L}=\frac{1}{\sqrt{n !}}\left(a_{-\vec{k}}^{\dagger}\right)^{n_{\vec{k}}}|0\rangle$ and $\left|n_{\vec{k}}\right\rangle_{R}=\frac{1}{\sqrt{n !}}\left(a_{\vec{k}}^{\dagger}\right)^{n_{\vec{k}}}|0\rangle$. We will not get into details of boundary states in more general CFTs in this paper.

\section{No real-space entanglement in boundary states}

We will now study the real-space entanglement of the quantum states defined by boundary states. Although boundary states are maximally entangled when the total Hilbert space is bipartitioned into the left and right-moving parts, as noted in the previous section, we will argue that boundary states have no real-space entanglement other than possible topological contribution.

\subsection{Boundary states from relevant perturbations}

Let us consider a relevant perturbation to a given 2d CFT Hamiltonian $H$ :

$$
H_{M}=H+M^{2-\Delta_{O}} \int d x O(x)
$$

where $O(x)$ is a relevant operator with the conformal dimension $\Delta_{O}$ and $M$ represents the mass scale of this massive deformation. We assume that under the renormalization group (RG) flow, the theory flows into a trivial IR theory which has no propagating degrees of freedom. We consider a quantum state $\left|\Omega_{M}\right\rangle$ defined as the ground state of the massive Hamiltonian $H_{M}$. In particular, we take $M \equiv 1 / \epsilon$ to be as large as the order of the UV cut off $\Lambda$, in which case the infinitesimally small quantity $\epsilon \sim 1 / \Lambda$ can be regarded as the lattice constant in a discretized lattice regularization. In this case, it is intuitively clear that real-space entanglement essentially vanishes for the scale much larger than $\epsilon$ since the Hamiltonian $H_{M}$, after a discretization, is approximated by the one with infinitely many disentangled points with the lattice constant $\epsilon$. Strictly speaking, there can still be finite quantum entanglement remained, which comes from topological order (including symmetry-protected topological phases - see below) as typical in the topological entanglement entropy [36, 37]. However this contribution is much smaller than the quantum entanglement which comes from the dynamics of CFTs. We will confirm this later explicitly in sections 3.3 for specific examples. Refer also to appendix A for calculations in a 
lattice fermion model. However, such finite contributions do not play any important role in our main arguments on classical gravity duals. (Indeed, such topological finite contribution is invisible in our holographic analysis in section 4.2.)

The above procedure of adding a massive perturbation to CFTs is closely related to the (global) quantum quenches, where the massive deformation is suddenly turned off at a given (initial) time [38]. Following the standard argument in this context, we then expect that the quantum state $\left|\Omega_{M}\right\rangle$ is given by a regularized boundary state:

$$
\left|\Omega_{M}\right\rangle=\hat{F}(\epsilon) \cdot|B\rangle,
$$

where $\hat{F}(\epsilon)$ is a regularization operation such that $\hat{F}(0)=1$. As in [38], for example, we can approximately choose this to be, $\hat{F}(\epsilon) \propto e^{-\epsilon H}$. Then the state (3.2) is described by a path-integral starting from the boundary for a (Euclidean) time period $\epsilon$ as in the upper picture in figure 1. This claim (3.2) can be understood by noting that the infinitely large massive deformation kills all dynamics, ending up with an empty theory and hence the massive deformation introduces a boundary beyond which there is no degree of freedom.

As a simple example, let us consider a free massive Dirac fermion in $(1+1) d$ [39]:

$$
H_{M}=\int d x\left[-i \psi^{\dagger} \sigma_{z} \partial_{x} \psi+m \psi^{\dagger} \sigma_{x} \psi\right]
$$

where $\psi=\left(\psi_{L}, \psi_{R}\right)^{T}$ is a two-component fermion field, and $m$ represents the mass term. The ground state of this Hamiltonian is given by

$$
\left|\Omega_{M}\right\rangle=\exp \left[\sum_{k>0} \frac{m}{\sqrt{m^{2}+k^{2}}+k}\left(\psi_{L k}^{\dagger} \psi_{R k}+\psi_{R-k}^{\dagger} \psi_{L-k}\right)\right]\left|G_{L}\right\rangle \otimes\left|G_{R}\right\rangle
$$

where $\psi_{L, R k}$ is the Fourier component of $\psi_{L, R}(x)$, and $\left|G_{L, R}\right\rangle$ is the Fock vacuum of the left- and right-moving sector in the massless limit, respectively. As we take $m \rightarrow \infty$ $\left(m /\left(v_{F} k\right) \rightarrow \infty\right),\left|\Omega_{M}\right\rangle$ reduces to the boundary states of the free massless fermion theory.

In summary, we obtained from the above argument the first explanation that boundary states do not have real entanglement by taking the cut off $\epsilon$ to zero. Obviously we can generalize this result to higher dimensions.

Note also that the boundary states we constructed in the above massive deformation should be the Cardy states $\left|C_{\alpha}\right\rangle$ as they describe physical boundaries. For example if we take $O(x)=\phi(x)^{2}$ in a free scalar field theory (i.e., the $\mathrm{U}(1)$ current algebra), it is clear that we get the Cardy state corresponding to the Dirichet boundary condition. It is a interesting future problem to find a general rule telling us which relevant perturbation in a bulk CFT leads to which boundary states.

\subsection{Correlation functions}

It is also helpful to study correlation functions for quantum states given by the boundary states. For this purpose consider the following $n$-point function of local operators at a particular time in arbitrary dimensions:

$$
\left\langle O\left(x_{1}\right) O\left(x_{2}\right) \cdots O\left(x_{n}\right)\right\rangle_{\text {Strip }}=\frac{\left\langle B\left|e^{-\epsilon H} O\left(x_{1}\right) O\left(x_{2}\right) \cdots O\left(x_{n}\right) e^{-\epsilon H}\right| B\right\rangle}{\left\langle B\left|e^{-2 \epsilon H}\right| B\right\rangle},
$$




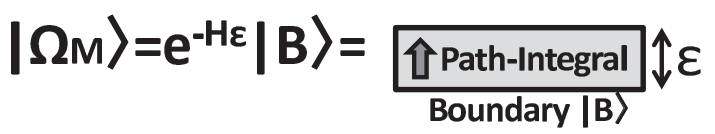

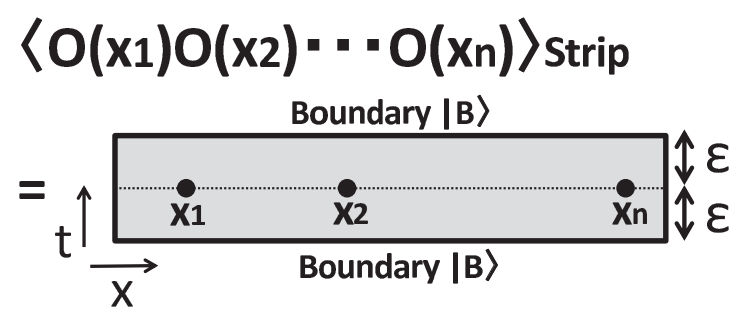

Figure 1. Sketches of path-integral representation of the regularized unentangled state $\left|\Omega_{M}\right\rangle$ and $n$ point functions $\left\langle O\left(x_{1}\right) O\left(x_{2}\right) \cdots O\left(x_{n}\right)\right\rangle_{\text {Strip }}$.

where $x_{1}, x_{2}, \cdots, x_{n}$ describe $n$ different spacial positions. In the path-integral formalism with the regularization $\epsilon$, this is evaluated as the path-integral over field configurations on the strip shape spacetime defined by the region $0 \leq t \leq 2 \epsilon$ with the $n$ operators inserted at the time $t=\epsilon$, as depicted in the lower picture of figure 1 . There are two spacelike boundaries at $t=0$ and $t=2 \epsilon$ which correspond to the boundary states.

In this setup it is obvious that in the limit $\epsilon \rightarrow 0$ with $x_{i} \neq x_{j}$ for all pairs $i \neq j$, the correlation function (3.5) is factorized into $n$ one point functions and does not depend on $x_{i}-x_{j}$. This argument again shows that boundary states do not essentially have real-space correlations. ${ }^{1}$

\subsection{A free field calculation of entanglement entropy of boundary states}

We now give an explicit estimate of the entanglement entropy $S_{A}$ for, as one of the simplest examples, a free massless Dirac fermion theory when $A$ is an interval. We employ the results obtained in [40] where the entanglement entropy under quantum quenches has been computed on a compact space. We assume the free Dirac fermion theory on a cylinder $(t, x)$ with the periodicity $x \sim x+2 \pi$. We choose the boundary state $|B\rangle$ to be either the Dirichlet or Neumann boundary condition (in terms of the scalar obtained from the bosonization of the $2 \mathrm{~d}$ massless Dirac fermion).

From the result in [40], we find the following entanglement entropy $S_{A}$ for the interval $A$ of length $l$ for both the Neumann and Dirichlet boundary conditions:

$$
S_{A}=\frac{1}{6} \log \frac{\left|\theta_{1}\left(\frac{l}{2 \pi} \mid \frac{2 i \epsilon}{\pi}\right)\right|^{2}\left|\theta_{1}\left(\frac{\epsilon}{\pi i} \mid \frac{2 i \epsilon}{\pi}\right)\right|^{2}}{\eta\left(\frac{2 i \epsilon}{\pi}\right)^{6} \cdot\left|\theta_{1}\left(\frac{\epsilon}{\pi i}-\frac{l}{2 \pi} \mid \frac{2 i \epsilon}{\pi}\right)\right|^{2}\left|\theta_{1}\left(\frac{\epsilon}{\pi i}+\frac{l}{2 \pi} \mid \frac{2 i \epsilon}{\pi}\right)\right|^{2} \cdot a^{2}},
$$

where $a=1 / \Lambda$ is the UV cut off (lattice spacing) of the free fermion theory. Note that in the continuum limit $a \rightarrow 0$ with $\epsilon$ kept finite, $S_{A}$ is logarithmically divergent, $S_{A} \sim \frac{1}{3} \log a^{-1}$,

\footnotetext{
${ }^{1}$ Notice that this argument, which is based on the correlation functions of local operators, can miss non-local correlation as typical in topological orders, mentioned in section 3.1. Such contribution, however, is not our main focus in this paper for our purpose of understanding holography.
} 
which is the well-known result in 2d CFTs [31] (remember the central charge is $c=1$ for the Dirac fermion). If we assume the parameter regime of our interest, $\epsilon \ll l$ and $\epsilon \ll 1$, the above entropy is simplified as

$$
S_{A}=\frac{1}{3} \log \frac{4 \epsilon}{\pi a} .
$$

Therefore we learned that if we take $\epsilon$ is of order of the UV cut off $a$, the logarithmically divergent term in $S_{A}$ disappears and thus $S_{A}$ is finite, i.e., $S_{A} \sim O(1)$. This shows that there is essentially no real-space entanglement in these boundary states.

It is also interesting to extend the above argument to the evaluation of $S_{A}$ for the Cardy states in general 2d CFTs. Again we assume that the subsystem $A$ is given by an interval of length $l$. By using the replica trick, we can represent $\operatorname{Tr}\left[\rho_{A}^{n}\right]$ as the path-integral on $0 \leq x \leq 2 \pi$ and $0 \leq t \leq 2 \epsilon$ with two twisted vertex operators at the ends of the interval $A$ [38]. In the limit $l \gg \epsilon$, these two vertex operators are far apart and thus are decoupled. The path-integral is then factorized into the two identical partition functions with single twisted operator, each of which can be conformally mapped to a disk partition function with the boundary condition specified by the Cardy state we consider. Since the disk partition function is proportional to the $g$-function [41], we obtain the following general result:

$$
S_{A}=\frac{c}{3} \log \frac{\epsilon}{a}+2 \log g+c_{1},
$$

where $c_{1}$ denotes an undetermined numerical constant, which does not depend on the boundary condition. Again this shows $S_{A} \sim O(1)$. It should be noted, however, that the above result (3.8) is quantitatively correct only when $\epsilon \gg a$. On the contrary, here we are interested in the case $\epsilon \sim a$ and therefore we cannot trust the precise value of the finite term in (3.8). Nevertheless, it is at least clear that the logarithmically divergent term of the entanglement entropy is absent for any boundary state, and this is enough to argue that boundary states essentially have no real-space entanglement.

On the other hand, boundary states in the limit $\epsilon \rightarrow 0$ can still have finite amount of the entanglement entropy, which deserves a further comment (although it does not affect our main arguments in this paper): while this constant term is non-universal in general, it has an important implication in the context of symmetry-protected topological phases in $(1+1)$ d. (For an overview of symmetry-protected topological phases, see [42-45]). It is known that all gapped phases in $(1+1)$ d can be continuously deformable (adiabatically connected) to each other. However, if some symmetry condition is imposed, two gapped phases may not be adiabatically connected, i.e., they are separated by a quantum phase transition. In particular, there may be a gapped phase which can be sharply (or "topologically") distinct from trivial gapped states (such as an atomic insulator). Such gapped phase is called a symmetry-protected phase. Examples of symmetry-protected topological phases in $(1+1) d$ include topological insulators, and the Haldane phase in integer spin chains [4650]. It has been argued that different SPT phases (respecting the same symmetry) can be distinguished by their entanglement spectrum, and, more precisely, by degeneracy in their entanglement spectrum [51]. For example, in the Haldane phase, the entanglement spectrum shows a characteristic double degeneracy for each entanglement eigenvalue, resulting 
in a $\log 2$ contribution to the entanglement entropy, whereas there is no such degeneracy in trivial phases. Thus, while there is no universal meaning in the constant entanglement entropy, if there is some symmetry condition, it may limit the minimal possible entanglement entropy, or the degeneracy of the entanglement spectrum. In appendix A, these points are illustrated by evaluating the finite entanglement entropy explicitly for a specific fermion model.

\section{Boundary states as holographic duals of trivial spacetimes}

In this section, as a main claim of this paper, we argue that boundary states in CFTs are dual to trivial spacetimes. By trivial spacetimes we mean those whose volume are of order of planck size, which vanishes upon taking the classical gravity limit.

\subsection{AdS/CFT and holographic entanglement entropy}

The AdS/CFT argues that the gravity on a pure AdS is equivalent to the ground state of a CFT defined on its boundary. This correspondence can be generalized to more general states in the CFT. If we pick up an arbitrary state in the CFT Hilbert space, it is dual to a gravity on a certain spacetime. The main question that we address below is what kind of gravitational spacetime is dual to CFT boundary states, for which we showed in the previous section that the real space entanglement essentially vanishes. As clear from our construction via relevant perturbations in section 3.1, a boundary state is a ground state of a Hamiltonian with an infinite mass. Therefore each (lattice) point in space where the CFT is defined is independent from the others, which leads no real space entanglement.

A hint on the nature of possible gravity dual of boundary states can be obtained from the tensor network construction of many-body wave functions. In the MERA description [12-15], a completely disentangled state is described by a trivial tensor network just consisting of one layer of lattice points of the original state. This is simply because we do not need to add any quantum entanglement. This should be contrasted with the MERA network for CFT ground states, where infinitely many layers of lattice points (tensors) are necessary, in order to reproduce quantum entanglement included in the ground state (for a brief review of MERA refer to section 5). By invoking the conjectured interpretation of AdS/CFT in terms of MERA [15], the MERA construction then tells us that boundary states are dual to trivial spacetimes.

We can also obtain this conclusion by looking at the entanglement entropy. In Einstein gravity, the holographic entanglement entropy [5-9] is given by

$$
S_{A}=\frac{\operatorname{Area}\left(\gamma_{A}\right)}{4 G_{N}},
$$

where $\gamma_{A}$ is the minimal area surface which ends on the boundary of subsystem $A$ (i.e. $\left.\partial \gamma_{A}=\partial A\right)$. In the presence of higher derivative corrections in string theory, the correct entropy is obtained by replacing the area term with curvature terms as

$$
S_{A}=\frac{1}{4 G_{N}} \int_{\gamma_{A}} \sqrt{g}\left(1+\lambda_{1} \alpha^{\prime} R+\ldots\right)
$$


where $\lambda_{1}$ is a parameter of the higher derivative correction [27-30]. Therefore, if we impose that the holographic entanglement entropy vanishes, $\gamma_{A}$ should be of zero size. Since for boundary states, we expect this for any choice of $A$, we can conclude that the total spacetime is vanishing.

Since we are considering spacetimes of zero size, we need to take into account quantum gravity corrections. For this we expect that the area and higher curvature terms are replaced with some quantum averages thereof, although there is no known precise formula which computes quantum corrections to the holographic entanglement entropy, except one loop corrections [52]. In this sense, it is possible that the spacetimes dual to boundary states have planck size volume, which can be regarded as trivial spacetimes from the viewpoint of classical gravity.

\subsection{An explicit construction in AdS/BCFT}

We can also study the gravity dual of boundary states by using the AdS/BCFT correspondence introduced in $[53,54]$. This is a construction of gravity dual of a CFT defined on a manifold with a boundary (BCFT). This formulation passes many non-trivial checks such as a holographic derivation of the $g$-theorem and computations of the entanglement entropy in BCFTs [53, 54].

As an illustration, we will concentrate on the two dimensional case. The generalization to higher dimensions is straightforward. According to the AdS/BCFT, the gravity dual of the state

$$
\rho \propto e^{-\epsilon H}|B\rangle\langle B| e^{-\epsilon H},
$$

which is equivalent to a $2 \mathrm{~d}$ CFT on a strip of width $2 \epsilon$, is given by a part of the Euclidean BTZ back hole. The metric of this (Euclidean) BTZ black hole is

$$
d s^{2}=R^{2}\left(\frac{h(z) d t^{2}}{z^{2}}+\frac{d z^{2}}{h(z) z^{2}}+\frac{d x^{2}}{z^{2}}\right), \quad h(z) \equiv 1-\frac{\pi^{2} z^{2}}{4 \epsilon^{2}},
$$

where $R$ is the AdS radius and the coordinates $(t, z, x)$ take values in the range $-2 \epsilon \leq t \leq$ $2 \epsilon, 0<z \leq 2 \epsilon / \pi,-\infty<x<\infty$. Note also that $t$ has the periodicity $4 \epsilon$. Then let us divide this spacetime described by (4.4) into two halves by a certain two-dimensional surface $Q$ which connects the two lines (extending in the $x$ direction) defined by $(t, z)=(0,0)$ and $(t, z)=(2 \epsilon, 0)$. Our gravity dual of the CFT on the strip is given by the spacetime which is surrounded both by $Q$ and by the boundary half circle (defined by $z=0$ and $0<t<2 \epsilon$ ). In other words, the gravity dual is given by cutting out the BTZ black hole along the surface $Q$.

As argued in [53, 54], this surface $Q$ can be found by solving the boundary equation of motion, which involves the extrinsic curvature. The shape of $Q$ is related to the $g$-function of the CFT. The symmetric case where $Q$ is simply given by the middle half circle $t=0$ and $t=2 \epsilon$ is equivalent to the construction by Hartman and Maldacena in [25], where the gravity dual of quantum quench is discussed.

Now if we consider $n$ point functions (3.5) in the gravity dual of a 2d CFT on the strip, it is obvious there is no geodesic which connects $x_{i}$ and $x_{j}(i \neq j)$ because of the presence 
of the boundary $Q$. Therefore there will be no correlations as long as $\left|x_{i}-x_{j}\right| \gg \epsilon$ in the approximation that the conformal dimensions of the local operators are very large.

We can also study the entanglement entropy. The holographic entanglement entropy is given by the area of minimal area surface divided by $4 G_{N}$ [5-9]. In the presence of spacetime boundary $Q$, the minimal surface can end on $Q$. Therefore if the subsystem $A$ is given by an interval with the length $l$, then the minimal surface is divided into two disconnected segments both of which connect the end points of $A$ to $Q$. When $\epsilon \gg a$, we can approximate the metric (4.4) by the pure $\mathrm{AdS}_{3}$, i.e., $h(z)=1$ and the surface $Q$ by two planes $t / z=$ const. and $(t-2 \epsilon) / z=$ const. By employing the holographic calculation of the $g$-function in $[53,54]$, we finally find

$$
S_{A} \simeq \frac{c}{3} \log \frac{2 \epsilon}{a}+2 \log g
$$

which agrees with (3.8). Here $a$ is the UV cut off of the CFT and this corresponds to the UV cut off $z>a$ in the gravity dual.

On the other hand, if we take $\epsilon$ to be of the same order as $a$, especially $\epsilon \leq \frac{\pi}{2} a$, then the gravity dual itself becomes empty after we impose the UV cut off $z>a$. Even if we assume $\epsilon>\frac{\pi}{2} a$, the whole spacetime disappear in the limit $\epsilon \rightarrow 0$, where the state (4.3) precisely coincides with the boundary state. Thus, we again reach our conclusion that boundary states are dual to trivial spacetimes. Note that our argument of AdS/BCFT is valid for classical gravity and neglects quantum gravity effects. Thus again it is possible that such spacetimes can have planck size volume.

It is useful to note that this holographic analysis shows $S_{A}=0$ for any $A$, though our general argument in the previous section shows that there can be a finite entanglement entropy if there is any topological order. This is consistent with holography as we do not expect any topological order in our classical gravity construction of AdS/BCFT. Indeed, it is shown in explicit examples that topological effects does not appear in the large $N$ limit [55].

\section{5 cMERA for general CFTs and boundary states}

In this section, by making use of our observation that boundary states in CFTs do not have real space entanglement, we propose a formulation of cMERA for general CFTs. We will first go through necessary ingredients of MERA [12-14] and its continuous formulation, cMERA [16], paying a special attention to a precise relation between coase-graining procedure in MERA and cMERA. We will also give a brief overview on the conjectured relation between MERA/cMERA and the AdS/CFT correspondence [15, 17]. For a review on MERA and tensor-network methods for many-body systems in general, refer to, e.g., [56]. The new cMERA construction using boundary states is applied to free massless scalar and free massless fermion theories in section 5.3, and produces valid results. In particular, for free massless fermion theories, the new cMERA construction improves upon the previous cMERA construction and reproduces the correct ground state for any energy scale. We also discuss the cMERA construction for generic 2d CFTs, which is consistent with the holographic interpretation. 


\subsection{MERA and cMERA}

MERA. MERA is a scheme for the real-space RG formulated in terms of wave functions. This should be contrasted to the more familiar method of the Wilsonian RG, where we consider the RG in terms of effective actions in momentum space. Suppose we aim to find the ground state of a complicated Hamiltonian, such as the one for a one-dimensional quantum spin system (spin chain). Following the spirit of the real-space RG in classical statistical systems, we consider to iteratively coarse-grain the spin chain by combining two quantum spins into one. Let us define a non-positive integer $u$ which counts the iterative steps of this coarse-graining. We describe the initial spin chain by $u=0$ and the first step of coarse-graining is denoted by $u=-1$. If we start with a spin chain with $N$ spins, after $|u|$ steps of coarse-graining the number of spins becomes $N \cdot 2^{u}$. In the end, it is reduced to a single spin after $\log _{2} N$ steps.

This coarse-graining procedure alone, however, does not give a good approximation to the correct ground state, in particular when the quantum spin chain does not have a mass gap; the amount of quantum entanglement included in the resulting wave function is much smaller than we wish, in that its entanglement entropy $S_{A}$ has a finite upper bound. On the other hand, $S_{A}$ in 2d CFTs increases logarithmically with respect to the size of $A$ [31]. To circumvent this problem in MERA, a special type of tensor, called disentanglers, are introduced. A disentangler is a unitary transformation which acts on nearest neighbor spins at each coarse-graining step, and, as we go through the coarse-graining procedure, it reduces bits of quantum entanglement of the original, highly entangled, ground state.

This MERA evolution, consisting of coarse-graining followed by disentanglers, can be run backward; starting from a single spin, we iteratively double the number of spins and, at each step, introduce quantum entanglement between adjacent spins by a unitary transformation, which is the (dis)entanglers. After many iterations, the MERA evolution reproduces the correct ground state. These steps are the basic construction of MERA. This formulation of $(1+1)$-d MERA can be generalized to higher dimensions in a straightforward way.

In [15], it was conjectured that the MERA formulation of CFTs is equivalent to the AdS/CFT correspondence by identifying the extra coordinate $u$ as the extra dimension. The coordinate $u$, which counts MERA steps, is expected to be related to $z$, the standard radial coordinate of Poincaré AdS, as $z=\epsilon \cdot 2^{-u}$, where the metric of Poincaré AdS is given by $d s^{2}=R^{2}\left(d z^{2}+d x^{2}\right) / z^{2}$, and $\epsilon$ is the UV cut off in the AdS/CFT. One important qualitative evidence for this conjecture comes from the fact that the estimation of the entanglement entropy in the MERA representation of quantum ground states is given by minimizing the intersections between bulk surfaces and entangling bonds, which looks analogous to the holographic entanglement entropy formula.

cMERA. In order to apply MERA to continuum field theories, the continuous MERA (cMERA) has been formulated [16]. We will consider cMERA for a $(d+1)$-d QFT and follow the convention in [17]. In cMERA, we start from the unentangled state $|\Omega\rangle$ (called the IR state) and introduce the entanglement at each length scale so that we can reproduce the correct state $\left|\Psi_{U V}\right\rangle$ (the UV state), which is typically a ground state of a given Hamiltonian. 
This construction is naturally understood as a continuous limit of MERA. Only apparent difference is that in cMERA the dimension of the Hilbert space (or the number of spins) does not change at each coarse-graining step. In other words all procedures in cMERA such as coarse-graining and disentangling are described by unitary transformations. It should be noted, however, that even in MERA, by adding dummy states at each coarse-graining step, one can keep the total number of spins. Refer to appendix B for an example of cMERA applied to free scalar fields.

We define a state $|\Psi(u)\rangle$ parameterized by the scale $u$. This state $|\Psi(u)\rangle$ is obtained by adding the entanglement for the momentum scale $k \leq \Lambda e^{u}$ to the unentangled state $|\Omega\rangle .^{2}$ The unentangled state (the IR state) $|\Omega\rangle$ is defined as a state without any real-space entanglement. ${ }^{3}$ As will be argued in a later subsection, the IR state is closely related to the boundary states of CFTs. We choose $\Lambda=1 / \epsilon$ to be the original UV cut off scale, where $\epsilon$ represents the lattice spacing. When $u=0$, the state $|\Psi(0)\rangle$ includes all the entanglement and coincides with the UV state of our interest (e.g. the ground state $|0\rangle$ of a given QFT). On the other hand, when $u=u_{I R}(=-\infty)$, the state does not include any entanglement and is given by the IR state $\left|\Psi\left(u_{I R}\right)\right\rangle=|\Omega\rangle$.

This procedure can explicitly be written as

$$
|\Psi(u)\rangle=P e^{-i \int_{u_{I R}}^{u}(K(s)+L) d s}|\Omega\rangle,
$$

where the symbol $P$ represents the path-ordering which puts all operators with smaller $u$ to the right. The operator $K(s)$ describes (dis)entangler which introduces necessary entanglement along the MERA evolution from $u=-\infty$ to $u=0$. The entangler $K(s)$ acts only on the momentum modes with $k \leq \Lambda$. For this purpose we introduce the cut off function $\Gamma(k / \Lambda)$ so that $\Gamma(x)=1$ for $0<x<1$ and $\Gamma(x)=0$ in other cases [16]. Schematically, we have

$$
K(u)=\int d^{d} k \Gamma(k / \Lambda) \cdot g(u, k) \cdot O_{k},
$$

for any $u$, where $O_{k}$ is a suitable operator with the energy scale $k$. We expect $\int d^{d} k O_{k}=$ $\int d^{d} x O(x)$, where $O(x)$ is a certain local operator in the CFT we consider. ${ }^{4}$ The function $g(u, k)$ depends on the theory and state of our interest and describes the strength of disentangling procedure. The operator $L$ in (5.1) is the non-relativistic scale transformation such that

$$
L|\Omega\rangle=0 .
$$

In the MERA language, this operator $L$ corresponds to the corase-graining procedure.

\footnotetext{
${ }^{2}$ We need to be careful for this interpretation. This bound $k \leq \Lambda e^{u}$ is based on the viewpoint from the UV state at $u=0$. From the viewpoint from the state $|\Psi(u)\rangle$, the cut off is always $k \leq \Lambda$. Refer to our explanations later.

${ }^{3}$ In massless theories (CFTs) we need to be careful. The IR state $|\Omega\rangle$ coincides with the unentangled state only if $k>\Lambda$. For $k<\Lambda$ we find $|\Omega\rangle$ is equal to the CFT vacuum $|0\rangle$. For more details see the example in appendix B.2 and (B.11).

${ }^{4}$ Note that $O_{k}$ is not a Fourier transformation of $O(x)$. Refer to an explicit example in appendix B.
} 
To establish a connection between cMERA and MERA for discrete lattice models more closely, we note, in the MERA picture, the effective lattice spacing is given by $\epsilon \cdot e^{-u}$ at the $(-u)$-th step. Therefore the effective momentum cut off is given by $\Lambda e^{u}$. However, in the description of cMERA (5.1) using $|\Psi(u)\rangle$, we rescale the length scale at each step such that the momentum cut off is always given by $k \leq \Lambda$ for any values of $u$. In other words, we always rescale the effective lattice spacing to be $\epsilon$ for any $u$ in the description by $|\Psi(u)\rangle$. In the holographic dual language, we are looking at the co-moving coordinate $x$ in the AdS space. This situation is depicted in the upper picture of figure 2 .

When applying cMERA for a CFT vacuum state $|0\rangle$, there is a relativistic scale transformation $L^{\prime}$ (i.e. the dilatation operator in the CFT). It should be noted that $L$ and $L^{\prime}$ are different because $L$ describes the spacial coarse-graining or the space-like scale transformation. The CFT vacuum $|0\rangle$ satisfies $L^{\prime}|0\rangle=0$ but $L|0\rangle$ is non-vanishing. For a cMERA for a CFT vacuum, we can find the relation

$$
K(u)+L=L^{\prime} \quad \text { for } \quad k \leq \Lambda .
$$

This means that the IR state $|\Omega\rangle$ is actually given by the vacuum $|0\rangle$ for $k \leq \Lambda$, which is a highly entangled state. While this may sound puzzling at first sight, this is simply an artifact of our rescaling procedure and nothing strange. In our construction of $|\Psi(u)\rangle$, the non-relativistic scale transformation $L$ is performed at each step and thus we disentangle degrees of freedom only for $k>\Lambda$ for any value of $u$. (On the other hand, if we consider mass gapped theories rather than CFTs, then this seemingly singular behavior will not arise. For more details of this issue, refer to (B.11) in the explicit construction of cMERA for free scalars in appendix B.)

Reformulated cMERA. In order to make a clearer connection to the MERA process, it is useful to define a rescaled state $|\Phi(u)\rangle$, which is defined by

$$
|\Phi(u)\rangle \equiv e^{i u L}|\Psi(u)\rangle=P e^{-i \int_{u_{I R}}^{u} \hat{K}(s) d s}|\Omega\rangle .
$$

Here $\hat{K}(s)$ is defined by the disentangler in the interaction picture

$$
\hat{K}(u)=e^{i u L} K(u) e^{-i u L} .
$$

Therefore we have the representation

$$
\hat{K}(u)=\int d^{d} k \Gamma\left(k e^{-u} / \Lambda\right) \cdot g\left(u, k e^{-u}\right) \cdot \tilde{O}_{k},
$$

where $\tilde{O}_{k}$ is defined as $e^{i u L} O_{k} e^{-i u L}=e^{d u} \tilde{O}_{k e^{u}}$. This means that the effective lattice spacing at the scale $u$ behaves like $\epsilon \cdot e^{-u}$ which agrees with the discrete MERA formulation.

To have a more concrete interpretation, let us assume a vacuum state for a free conformal field theory, where $\hat{K}(u)$ is Gaussian:

$$
\hat{K}(u) \propto \int d^{d} k \Gamma\left(k e^{-u} / \Lambda\right) b_{k}^{\dagger} b_{-k}^{\dagger}
$$




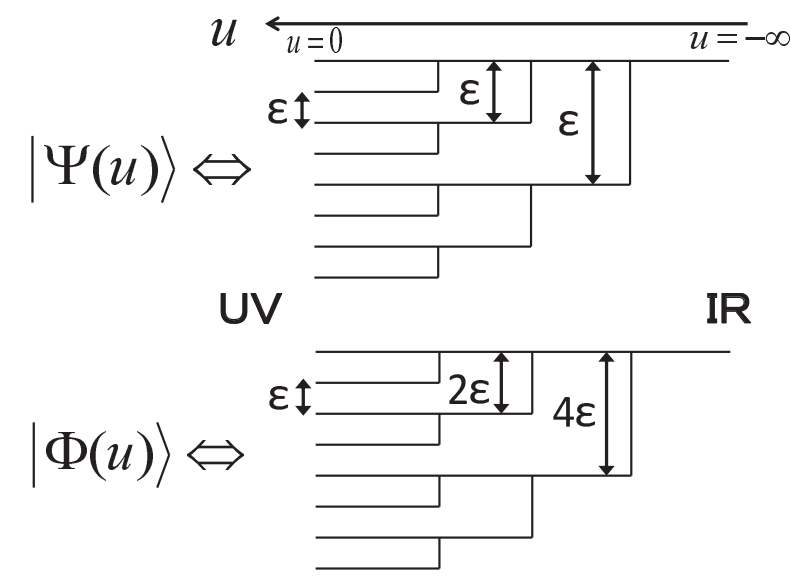

Figure 2. Interpretations of two different cMERA states $|\Psi(u)\rangle$ and $|\Phi(u)\rangle$ in terms of MERA for discretized lattice models. In the former state, we always rescale the distance between two adjacent lattice points to be $\epsilon$, whereas in the latter state we do not rescale.

where $b_{k}^{\dagger}$ is a creation operator of free field with momentum $k$ (see appendix B). This can be rewritten by defining a position space creation operator $b_{x}^{\dagger} \sim \int d^{d} k e^{i k x} b_{k}^{\dagger}$ as

$$
\begin{aligned}
\hat{K}(u) & \propto \int d^{d} x d^{d} y b_{x}^{\dagger} b_{y}^{\dagger} f(x-y), \\
\text { where } \quad f(x-y) & =\int d^{d} k e^{i k(x-y)} \Gamma\left(k e^{-u} / \Lambda\right) .
\end{aligned}
$$

We find that the function $f(x-y)$ takes non-trivial values only when $|x-y| \leq \epsilon \cdot e^{-u}$. ${ }^{5}$ This agrees with the discrete MERA formulation. Refer to the lower picture in figure 2.

\subsection{IR states for general CFTs}

In the above subsection we reviewed the idea of cMERA formulation. However, if we are to construct cMERA for generic CFTs, we need to understand how to construct the IR state $|\Omega\rangle$. In this subsection we discuss a general construction of the IR state. We focus on the formulation based on the state $|\Phi(u)\rangle$ below. However we can always relate results from $|\Phi(u)\rangle$ to $|\Psi(u)\rangle$ via the $L$ rescaling. Our claims in this subsection can be confirmed explicitly in the free scalar example of appendix B.

To realize a disentangled IR state, as in (3.1) we consider a relevant deformation of the original $d+1$ dimensional CFT Hamiltonian $H$ :

$$
H_{M}=H+M^{(d+1)-\Delta_{O}} \int d^{d} x O(x),
$$

where $O(x)$ is a relevant operator with the conformal dimension $\Delta_{O}$. The infinitely large parameter $M$ represents the mass scale of this massive deformation, which is identified

\footnotetext{
${ }^{5}$ This observation is immediately understood by approximating the cutoff function by the gaussian $\Gamma(x) \sim e^{-x^{2}}$. However, the same conclusion can also be obtained for our original cutoff function $\Gamma(x)=$ $1-\theta(|x|-1)$.
} 
with the UV cut off (or lattice spacing) as $M=1 / \epsilon$. We need to pick up an operator $O(x)$ which leads to a trivial IR fixed point under the RG flow. If we consider cMERA for a mass gapped QFT defined by a relevant perturbation of a CFT, we can choose $O(x)$ to be this relevant perturbation operator and our argument below still hold.

We now consider the ground state of $H_{M}$, denoted by $\left|\Omega_{M}\right\rangle$, and define it to be the IR state of cMERA, $|\Omega\rangle$. We propose that this is a general construction of IR states in any CFTs. Even though $H$ has the relativistic scale symmetry (dilatation) generated by $L^{\prime}$ such that $\left[L^{\prime}, H\right]=i H$, the mass deformed Hamiltonian $H_{M}$ does not have such a symmetry. Instead it is invariant under a non-relativistic scale transformation $L$ such that $\left[H_{M}, L\right]=0$. We can define $L$ such that the IR state $|\Omega\rangle$, which is the ground state of $H_{M}$, is invariant under $L$ as $L|\Omega\rangle=0$.

As discussed below (3.1), the IR state $|\Omega\rangle$ defined as the ground state of $H_{M}$ is given by a regularized boundary state as in (3.2). Especially in the limit $M \rightarrow \infty$ (or $\epsilon \rightarrow 0$ ), it should precisely approach to the boundary state $|B\rangle$. Also it is useful to notice that the boundary state has the relativistic scale symmetry such that $L^{\prime}|B\rangle=0$. Moreover, note that, in the case of $2 \mathrm{~d}$ CFTs in particular, these boundary states should be regarded as the Cardy states instead of the Ishibashi states because the infinitely large relevant perturbation leads to a physical boundary in the time direction.

\subsection{A general ansatz for cMERA}

Following the above argument, one of the simplest ansatz of the IR state is

$$
|\Omega\rangle=\mathcal{N} e^{-\epsilon H}|B\rangle
$$

where $\mathcal{N}$ is a normalization constant. This is equivalent to the approximation used for quantum quenches in [38]. Since boundary states can support topological entanglement, we expect this ansatz is valid for a wide class of vacuum states, including those with topological entanglement. We now apply this approximation to cMERA for CFTs. We will employ the rescaled formalism (5.5).

For this purpose, it is convenient to regard the UV cutoff $\Lambda$ in the disentangler as an artificial cutoff for calculations which we can make much larger than the lattice spacing, $\Lambda \gg M=1 / \epsilon$ : by making $\Lambda$ arbitrarily large, we can make the MERA wave function for $k<1 / \epsilon$ as close as possible to the true (ground) state. (We are interested only in physics below the energy scale $M$.) Finally we can determine the disentangler $\hat{K}(u)$ from the relation (5.5). In order to get a simple analytical control, we study the free scalar CFTs and the free fermion CFT in $2 \mathrm{~d}$ below.

\subsubsection{Free massless scalar CFTs}

As the first example of a CFT, consider a free massless scalar field in any dimension. We choose the disentangler in the following form (see also (B.5) in appendix B)

$$
\hat{K}(u)=\frac{i}{2} \int d^{d} k \Gamma\left(k e^{-u} / \Lambda\right)\left(g(u) a_{k}^{\dagger} a_{-k}^{\dagger}-g^{*}(u) a_{k} a_{-k}\right) .
$$


The strength of disentangling procedure at energy scale $\Lambda e^{u}$ is estimated by $|g(u)|$. For our purpose we can assume that $g(u)$ is real-valued. From (5.5) with the ansatz (5.11), we see that $g(u)$ must satisfies

$$
2 \int_{-\infty}^{0} d u \Gamma\left(|k| e^{-u} / \Lambda\right) g(u)=\log \left(1+e^{-2 \epsilon|k|}\right)-\log \left(1-e^{-2 \epsilon|k|}\right) .
$$

This relation is obtained from the Bogoliubov transformation description generally formulated in section 3.2 of [17]. Assuming $\Lambda \epsilon \gg 1$, we can solve this as follows

$$
g(u)=\frac{e^{\tilde{u}}}{\sinh \left(2 e^{\tilde{u}}\right)},
$$

where we defined $\tilde{u}$ by shifting $u$ as follows

$$
\tilde{u}=\log (\Lambda \epsilon)+u
$$

Note that in the physically interesting range $-\infty<\tilde{u}<0$, the function $g(u)$ takes $O(1)$ non-vanishing values. This qualitatively tells us that the disentangling procedure continues for any $u$, which is consistent with the fact that we are considering a CFT.

\subsubsection{Free massless fermion CFT in two dimensions}

As another example, let us consider the free massless Dirac fermion in two dimensions. We introduce the disentangler as (here we follow the notations in ref. [17].)

$$
\hat{K}(u)=i \int d k\left[g_{k}(u) \psi_{1}^{\dagger}(k) \psi_{2}(k)+g_{k}^{*}(u) \psi_{1}(k) \psi_{2}^{\dagger}(k)\right],
$$

where we defined

$$
g_{k}(u)=g(u) \Gamma\left(|k| e^{-u} / \Lambda\right) \frac{k e^{-u}}{\Lambda} .
$$

Again we can assume $g(u)$ is real-valued. With the IR state (5.11), the relation (5.5) leads to the condition

$$
\cos \left[\frac{k e^{-u}}{\Lambda} \int_{-\infty}^{0} d u \Gamma\left(k e^{-u} / \Lambda\right) g(u)\right]=\frac{1}{\sqrt{1+e^{-4|k| \epsilon}}} .
$$

Solving this equation, we obtain

$$
g(u)=\frac{e^{\tilde{u}}}{\cosh \left(2 e^{\tilde{u}}\right)}+\arccos \left(\frac{1}{\sqrt{1+e^{-4 e^{\tilde{u}}}}}\right),
$$

where $\tilde{u}$ is defined as before (5.15). Again, in the physically interesting range $-\infty<$ $\tilde{u}<0$, the function $g(u)$ takes $O(1)$ non-vanishing values. This approximated solution improves the construction of cMERA for the free fermion in [16]. The difference between our construction and the one in [16] is the choice of the IR state. As compared to our choice 5.11, the IR state in ref. [16] is similar, but without the regularization factor $e^{-\epsilon H}$, and hence the construction in ref. [16] can only be applied to the low energy region $k \epsilon \ll 1$. 


\subsection{3 cMERA for general 2d CFTs}

To demonstrate further the use of boundary states as IR states in cMERA, let us now consider generic 2d CFTs. We argue that a good cMERA ansatz is given by

$$
|\Phi(u)\rangle=\mathcal{N}_{u} \cdot e^{-\beta(u) H}|B\rangle, \quad \beta(u) \equiv \frac{\epsilon}{\left(e^{u / 2}-1\right)^{2}},
$$

where $\mathcal{N}_{u}$ is the normalization constant such that $\langle\Phi(u) \mid \Phi(u)\rangle=1$. Indeed this reproduces our IR state (5.11) in the IR limit $u \rightarrow-\infty$. On the other hand, in the UV limit $u \rightarrow 0$ we find that the state (5.20) approaches to the vacuum $|0\rangle$ because $\beta(u)$ gets positively divergent.

While (5.20) correctly interpolates the IR state (boundary state) and the UV state (the ground state), it is not written in the form compliant to (5.5), and hence, it is rather cumbersome to extract the form of disentangler from (5.20). Nevertheless, we can estimate the strength of the disentangler by computing quantum information metric, which also has a close connection to the metric of the gravity dual in the extra dimension [17]. The information metric is defined from the inner product between two states with infinitesimally different values of $u$ :

$$
1-|\langle\Phi(u) \mid \Phi(u+d u)\rangle|=G_{u u} d u^{2} .
$$

For our state (5.20) we can estimate the inner product as follows

$$
|\langle\Phi(u) \mid \Phi(u+d u)\rangle| \propto \int_{0}^{\infty} d E e^{2 \pi \sqrt{\frac{c E}{12 L}}} \cdot e^{-\frac{(\beta(u)+\beta(u+d u)) E}{L}} \simeq e^{\frac{\pi^{2} c L}{6(\beta(u)+\beta(u+d u))}}
$$

where $L$ is the total size of space where the $2 \mathrm{~d}$ CFT is defined. $E$ is the dimensionless energy defined by the eigenvalues of $H / L$. We employed an approximation using the Cardy formula [57] of the degeneracy of states in the chiral CFT, which is justified as we take $\epsilon$ to be infinitesimally small. Here, in applying the Cardy formula, note that in BCFTs the state degeneracy is the same as chiral CFTs with the energy divided by half. The overall normalization in (5.22) is fixed simply by requiring $\langle\Phi(u) \mid \Phi(u)\rangle=\langle\Phi(u+d u) \mid \Phi(u+d u)\rangle=1$. From (5.22) the information metric in the cMERA for general 2d CFTs is evaluated as

$$
G_{u u}=\frac{\pi^{2} c L}{24 \epsilon} \cdot \frac{\left(\partial_{u} \beta(u)\right)^{2}}{\beta(u)^{3}}=\frac{\pi^{2} c L}{24 \epsilon} e^{u} .
$$

This agrees with the free theory calculation in [17], where $G_{u u}$ for a $(d+1)$-d free field theory is given by $\frac{V_{d}}{\epsilon^{d}} e^{d u}$ times a constant proportional to the number of free fields $\left(V_{d}\right.$ is the volume of the $d$ dimensional space). This form (5.23) also agrees with the AdS metric using the holographic dictionary in [17]. Summarizing, by identifying the IR state as a boundary state, we have obtained the general construction cMERA for any $2 \mathrm{~d}$ CFTs in a way consistent with the holographic interpretation.

\section{Conclusions and discussion}

In this paper, we have studied quantum entanglement included in boundary states in CFTs. Boundary states have been playing an important role in boundary critical phenomena in 
condensed matter physics, and also in describing D-branes in string theory. Our findings in this paper add more importance to boundary states from a quantum information theoretic viewpoint.

In particular, we studied real-space entanglement of boundary states, which can be measured by the entanglement entropy when we spatially divide the system into two subsystems. We gave several independent field theoretic as well as holographic arguments, all of which show that there is no real-space entanglement coming from local degrees of freedom in boundary states. While this may sound counterintuitive, given that the left- and right-moving sectors are maximally entanglement in boundary states, (almost) vanishing entanglement in boundary states may be understood from entanglement monogamy, one of the basic properties of quantum entanglement; if two qubits A and B are maximally entangled, they cannot be entangled at all with a third qubit $\mathrm{C}$.

From the holographic point of view, the above result suggests that boundary states are dual to trivial spacetimes of zero spacetime volume, as follows from the holographic entanglement entropy. (We should however keep in mind that in such a situation, we have substantial quantum gravity effects.)

The vanishing entanglement in boundary states also has an implication and application in MERA which we believe captures fundamental aspects of the mechanism of the AdS/CFT correspondence. In the MERA construction, starting from a unentangled state (IR state), quantum entanglement is added at each length scale from IR to UV to reproduce a highly entangled state of our interest, such as the ground state of a complex many-body Hamiltonian. This procedure has a holographic interpretation in which, starting from a trivial spacetime, gradually adding pieces of spacetime eventually leads to a gravitational theory in an extended spacetime. By using a continuum limit of MERA (cMERA), we found that the IR unentangled state is given by a (regularized) boundary state. Owing to this identification, we have obtained the general construction cMERA for any 2d CFTs in a way consistent with the holographic interpretation. We also confirmed that this new construction leads to sensible results for free massless scalar field theories. For free massless fermion theories our general construction improves a problem known before and gives a complete cMERA construction valid for any energy scale.

Before closing, a few comments are in order:

(i) Strictly speaking, the real space quantum entanglement in boundary states is not always completely vanishing, but can have topological contributions as mentioned in section 3 and explicitly demonstrated in appendix A. However these contributions are much smaller than the dynamical contributions in the ground state of CFTs because the latter are UV divergent quantities, while the former are $O(1)$ finite quantities. In this paper we neglected such topological contributions as they do not play an important role in the holography with the classical gravity limit. Indeed, such a small entanglement entropy corresponds to a planck unit surface area and cannot be distinguished from a trivial point within the Einstein gravity. Nevertheless, we can still speculate that a boundary state with topological order can be dual to a pointlike object with a brane source, motivated by the holographic setup of the fractional quantum Hall effect discussed in [55]. 
(ii) For a given CFT, there are various choices of boundary states. In our formulation they correspond to various choices of the relevant deformation (5.10). This means, in fact, that the choice of the IR state $|\Omega\rangle$ is not unique. It is an important future problem to find a general rule as to which relevant deformation leads to which boundary state. Related to this non-uniqueness, it would be interesting to speculate upon formulating the cMERA for the canonical examples of the AdS/CFT, so that it is dual to taking slices in the AdS spacetime. We then need to preserve the R-symmetry, which is dual to the symmetry of internal manifolds such as $S^{5}$ in $A d S \times S^{5}$. In the setup of a global AdS in dimensions higher than three, it is easy to identify the relevant deformation in the dual CFT on the compact spacetime with the conformal mass terms of the CFT $\sim \int R \phi^{2}$, which manifestly preserves the R-symmetry. It is natural that for the Poincare AdS setup, we can still employ the same form of mass terms to define the cMERA for a CFT in a non-compact space. It will be important to understand a systematic relation between the choices of boundary states and their gravity duals.

(iii) Finally, in order to relate the cMERA formulation to the AdS/CFT, we expect that taking the large- $N$ or large central charge limit of CFTs is necessary. It is therefore an important future task to study the behavior of boundary states in this limit. Roughly speaking, we can regard the unitary transformation of a vacuum state into a boundary state (2.1) as a generalized version of the Bogoliubov transformation. This is manifest and exact in the example of the $\mathrm{U}(1)$ current algebra in (2.7). It is also curious to note that the Gaussian form of (2.7) is analogous to the double trace deformations which appear in the analysis of the holographic RG $[58,59]$.

\section{Acknowledgments}

We would like to thank Gil Young Cho, Pedro Lopes, Shunji Matsuura, Masahiro Nozaki, Tokiro Numasawa, Hirosi Ooguri, Xiaoliang Qi, Mark Van Raamsdonk, Mukund Rangamani and Masaaki Shigemori for useful discussions. TT is very grateful to the workshop 'Quantum Information Physics (YQIP2014)' at Yukawa Institute for Theoretical Physics, Kyoto University and the workshop 'Quantum Information in Quantum Gravity' at University of British Columbia in Vancouver for giving opportunities to present a part of this work in very stimulating atmospheres. TT is supported by JSPS Grant-in-Aid for Scientific Research (B) No.25287058 and JSPS Grant-in-Aid for Challenging Exploratory Research No.24654057. TT is also supported by World Premier International Research Center Initiative (WPI Initiative) from the Japan Ministry of Education, Culture, Sports, Science and Technology (MEXT). SR is supported by Alfred P. Sloan foundation.

\section{A A lattice model calculation of entanglement entropy of boundary states}

In this appendix, we use an example of a $(1+1) d$ lattice free fermion model to demonstrate: (i) inside its gapped phases, the ground state can be expressed as a boundary state; (ii) the entanglement entropy of the ground state is finite and constant for a subsystem of 
arbitrary size; (iii) the finite entanglement entropy is non-universal in general, but takes a universal value when a symmetry is imposed. Consider a tight-binding Hamiltonian (the Su-Schrieffer-Heeger ( $\mathrm{SSH})$ model):

$$
H=t_{1} \sum_{i}\left(c_{A i}^{\dagger} c_{B i}+\text { h.c. }\right)-t_{2} \sum_{i}\left(c_{B i}^{\dagger} c_{A i+1}+\text { h.c. }\right)+\mu_{s} \sum_{i}\left(c_{A i}^{\dagger} c_{A i}-c_{B i}^{\dagger} c_{B i}\right),
$$

where $\left(c_{A i}, c_{B i}\right)$ represents two-flavors of fermion annihilation operators defined at site $i$ on a $1 \mathrm{~d}$ lattice, and $t_{1}, t_{2}, \mu_{s}$ are real parameters. We assume $t_{1} \geq 0$ and $t_{2} \geq 0$ henceforth. When $\mu_{s}=0$, the Hamiltonian has chiral symmetry (sublattice symmetry); there is a matrix element connecting sites in A and B sublattice, but there is no matrix connection sites within the same sublattice. With this symmetry, it is known that the phase with $t_{1}>t_{2}$ and $t_{2}>t_{1}$ are topologically distinct and are always separated by a quantum critical point [61-63]. (In the above model, there is a phase transition when $t_{1}=t_{2}$.) Ground states in the phase $t_{1}>t_{2}$ are adiabatically connected to an atomic insulator (a collection of decoupled lattice sites) at $t_{1}>0$ and $t_{2}=0$, i.e., the system is in the "chiral topological insulator" phase. On the other hand, ground states in the phase $t_{2}>t_{1}$ are topologically distinct from topologically trivial, atomic insulators, once chiral symmetry is imposed [60]. Near the critical point, the SSH model reduces to the continuum Dirac Hamiltonian when expanded around $k=0$ with $t_{1}-t_{2}$ playing the role of the mass $m$.

The ground states of the SSH model can be explicitly constructed as follows. In momentum space, the Hamiltonian is written as $H=\sum_{k} \Psi^{\dagger}(k) \mathcal{H}(k) \Psi(k)$, where

$$
\Psi(k)=\left[\begin{array}{c}
c_{k} \\
d_{k}
\end{array}\right], \quad \mathcal{H}(k)=\vec{R}(k) \cdot \vec{\sigma}, \quad \vec{R}(k)=\left[\begin{array}{c}
t_{1}-t_{2} \cos k \\
-\mu_{s} \\
t_{2} \sin k
\end{array}\right],
$$

and $-\pi \leq k<\pi$; we assume the theory is defined on a finite ring and the fermions obey a suitable boundary conditions, e.g., antiperiodic boundary condition; we have rotated the Pauli matrices as $\left(\sigma_{x}, \sigma_{y}, \sigma_{z}\right) \rightarrow\left(\sigma_{x}, \sigma_{z},-\sigma_{y}\right)$ for convenience; the field operator $\Psi(k)$ represents the fermionic annihilation operator in this rotated basis. This Hamiltonian can be split as $H=H_{L}+H_{R}+H_{L R}$, where $H_{L}$ and $H_{R}$ represent lattice analogues of the leftand right-moving parts of the continuum Dirac Hamiltonian and are given by

$$
H_{L}=t_{2} \sum_{k} \sin (k) c_{k}^{\dagger} c_{k}, \quad H_{R}=-t_{2} \sum_{k} \sin (k) d_{k}^{\dagger} d_{k},
$$

whereas $H_{L R}$ plays the role of the mass and is given by

$$
H_{L R}=\sum_{k}\left(t_{1}-t_{2} \cos k+i \mu_{s}\right) c_{k}^{\dagger} d_{k}+\text { h.c. }
$$

By defining the Fock vacuum of $H_{L}$ and $H_{R}$ as $\left|G_{L}\right\rangle$ and $\left|G_{R}\right\rangle$, respectively, the (unnormalized) ground state of the total Hamiltonian is

$$
|\Omega\rangle=\exp \left[\sum_{k>0}\left(\frac{v_{k}}{u_{k}} c_{k}^{\dagger} d_{k}+\frac{u_{-k}}{v_{-k}} d_{-k}^{\dagger} c_{-k}\right)\right]\left|G_{L}\right\rangle \otimes\left|G_{R}\right\rangle,
$$


where $u_{k}$ and $b_{k}$ are given in terms of $\vec{R}(k)$ by

$$
\left(\begin{array}{c}
u_{k} \\
v_{k}
\end{array}\right)=\frac{R+R_{3}}{\sqrt{2 R\left(R+R_{3}\right)}}\left(\begin{array}{c}
R+R_{3} \\
R_{1}-i R_{2}
\end{array}\right), \quad R(k) \equiv|\vec{R}(k)| .
$$

Setting $\mu_{s}=0$, we are interested in the ground states deep inside the two phases (i.e., far away from the critical point):

$$
|G\rangle= \begin{cases}\exp \left[-\sum_{k>0}\left(c_{k}^{\dagger} d_{k}+d_{-k}^{\dagger} c_{-k}\right)\right]\left|G_{L}\right\rangle \otimes\left|G_{R}\right\rangle, & t_{1}>0, t_{2}=0 \\ \exp \left[+\sum_{k>0} \frac{\cos k}{1+\sin k}\left(c_{k}^{\dagger} d_{k}+d_{-k}^{\dagger} c_{-k}\right)\right]\left|G_{L}\right\rangle \otimes\left|G_{R}\right\rangle, & t_{1}=0, t_{2}>0\end{cases}
$$

The ground state for $t_{1}>0, t_{2}=0$ represents an atomic insulator where each site is completely isolated in the lattice model, and hence $S_{A}=0$ for any bipartition of the $1 \mathrm{~d}$ lattice. Taking "the continuum limit", where we regard $c, d$ as $\psi_{L}, \psi_{R}$, respectively, we reproduce the boundary state of the continuum massless Dirac theory.

The ground state for $t_{1}=0, t_{2}>0$ represents a topological insulator with ultra short correlation length. By making the transition from the lattice fermion operator into the continuum Dirac fields as $c \rightarrow \psi_{L}$ and $d \rightarrow \psi_{R}$, and by taking the low energy limit $k \rightarrow 0$, the ground state is again given by the boundary state. While the correlation length of the ground state for $t_{1}=0, t_{2}>0$ is as short as the lattice constant, the ground state has non-zero entanglement entropy. Taking the subregion $A$ as an interval of arbitrary size, we obtained a finite entanglement entropy $S_{A}=2 \log 2$. (See ref. [60] and the numerical calculation below.)

The finite entanglement comes from the UV physics, in that in the IR limit $k \rightarrow 0$ the ground states for topologically trivial and non-trivial phases show no difference (except an overall minus sign in the exponential, which can be changed by simply reversing the sign of the hopping parameter in the SSH model and hence is immaterial.) This finite, constant contribution to the entropy, $2 \log 2$, means that the entanglement spectrum defined for the subregion $A$ of arbitrary size is at least doubly degenerate. This double degeneracy is "symmetry-protected" and true for any ground state as far as the system is in the topological insulator phase. Once we break the symmetry, i.e., chiral symmetry, by introducing non-zero $\mu_{s}$, say, the symmetry protection is lost and the size-independent part of the entanglement entropy can take any value depending on microscopic details.

In figure 3, a numerical calculation of the time-evolution of the entanglement entropy $S_{A}$ after a quantum quench into the critical theory defined by $t_{1}=t_{2}$ and $m_{2}=0$ is presented, which demonstrates, at $t=0, S_{A}=0$ for the topologically trivial ground state whereas $S_{A}=2 \log 2$ for the topological insulator ground state. On the other hand, for $t$ sufficiently larger than some threshold, $t_{*}$, and for $t$ sufficiently smaller than the length of the region $A, l$, the time evolution of the entanglement entropy is the one predicted from the CFT calculation [38, 40]. As mentioned above, the two possible values of $S_{A}$ at $t=0$ are quite robust, as far as chiral symmetry is preserved. For example, changing the 


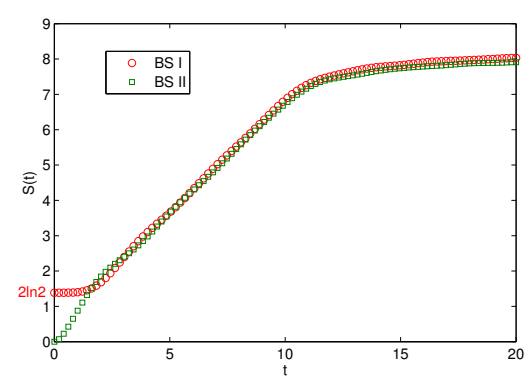

(a)

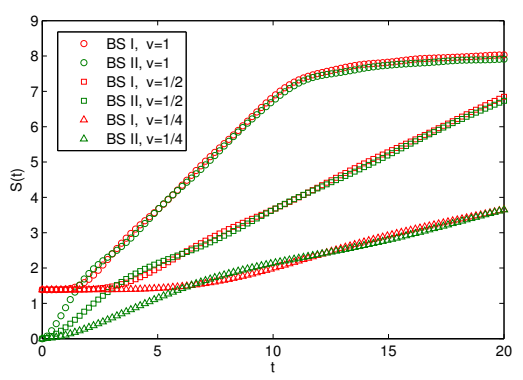

(b)

Figure 3. The numerically calculated time-evolution of the entanglement entropy, $S_{A}(t)$ of the SSH model, after a global quench into the critical state for different boundary states (denoted by "BS I" and "BS II", corresponding to the case of $t_{1}=0, t_{2}>0$ and $t_{1}>0, t_{2}=0$ in (A.7), respectively). We choose the size of the subregion $A l=10$ and the total system size $L=200$ (both measured in the lattice constant). (a) $S(t)$ as a function of time $t$. (b) The velocity $(v)$ dependence of $S_{A}(t)$.

velocity of the fermion mode (denoted by $v$ in figure 3, which is controlled by the hopping parameter $t_{2}$ in the lattice model) changes the threshold value $t_{*}$, while the value of $S_{A}(0)$ remains unaffected.

\section{B Example: cMERA for free scalar field theory}

Consider the free scalar field theory in $d+1$ dimensions (with mass $m$ ), which is one of the simplest examples of cMERA $[16,17]$. The time and space coordinates are denoted by $t$ and $x$. The energy and the momentum are written as $\epsilon$ and $k$, respectively, and the dispersion relation is given by $\epsilon_{k}=\sqrt{k^{2}+m^{2}}$. The Hamiltonian of this theory is defined by

$$
H=\frac{1}{2} \int d^{d} k\left[\pi(k) \pi(-k)+\epsilon_{k}^{2} \phi(k) \phi(-k)\right] .
$$

The creation and annihilation operators, $a_{k}^{\dagger}$ and $a_{k}$, can be introduced as follows

$$
\phi(k)=\frac{a_{k}+a_{-k}^{\dagger}}{\sqrt{2 \epsilon_{k}}}, \quad \pi(k)=\sqrt{2 \epsilon_{k}}\left(\frac{a_{k}-a_{-k}^{\dagger}}{2 i}\right) .
$$

They satisfy the canonical commutation relation $\left[a_{k}, a_{k^{\prime}}^{\dagger}\right]=\delta^{d}\left(k-k^{\prime}\right)$.

\section{B.1 Construction of cMERA}

In the IR limit, the Hamiltonian becomes infinitely many copies of decoupled harmonic oscillators located at each lattice point. We can define an unentangled state $\left|\Omega_{M_{m}}\right\rangle$ as the ground state for this harmonic oscillators, i.e., $a_{x}\left|\Omega_{M_{m}}\right\rangle=0$. Here $a_{x}=\sqrt{M_{m}} \phi(x)+$ $i \pi(x) / \sqrt{M_{m}}$ is the annihilation operator in real-space, and $M_{m}$ is the energy scale below which the disentanglement takes place. In other words, we assume the system is discretized into a lattice with lattice constant $\sim 1 / M_{m}$. In momentum space, this condition is equivalent to

$$
\left(\alpha_{k} a_{k}+\beta_{k} a_{-k}^{\dagger}\right)\left|\Omega_{M_{m}}\right\rangle=0
$$


where

$$
\begin{aligned}
\alpha_{k} & =\frac{1}{2}\left(\sqrt{\frac{M_{m}}{\epsilon_{k}}}+\sqrt{\frac{\epsilon_{k}}{M_{m}}}\right), \quad \beta_{k}=\frac{1}{2}\left(\sqrt{\frac{M_{m}}{\epsilon_{k}}}-\sqrt{\frac{\epsilon_{k}}{M_{m}}}\right), \\
M_{m} & =\sqrt{\Lambda^{2}+m^{2}} .
\end{aligned}
$$

Since we are dealing with a non-interacting theory, we assume the disentangler $\hat{K}(5.7)$ is "Gaussian" and takes the following form

$$
\hat{K}(u)=\frac{i}{2} \int d^{d} k \Gamma\left(k e^{-u} / \Lambda\right)\left(g(u) a_{k}^{\dagger} a_{-k}^{\dagger}-g^{*}(u) a_{k} a_{-k}\right),
$$

where $\Gamma(x)$ is a cut off function such that $\Gamma(x)=1$ when $x \leq 1$ and $\Gamma(x)=0$ for $x>1$. Indeed, by requiring that the UV state to be the CFT vacuum

$$
|\Phi(0)\rangle=|0\rangle
$$

(or equally $|\Psi(0)\rangle=|0\rangle$ ), we find the disentangler which reproduces the exact ground state as $[16]$

$$
g(u)=g^{*}(u)=\frac{1}{2} \cdot \frac{e^{2 u}}{e^{2 u}+m^{2} / \Lambda^{2}} .
$$

\section{B.2 Scale transformation in massless scalar}

The non-relativistic and relativistic scale transformation in the free boson theory are defined as follows [16]

$$
\begin{aligned}
L & =-\frac{1}{2} \int d^{d} x\left[\pi(x) \vec{x} \cdot \vec{\nabla}_{x} \phi(x)+\vec{x} \cdot \vec{\nabla}_{x} \phi(x) \pi(x)+\frac{d}{2} \phi(x) \pi(x)+\frac{d}{2} \pi(x) \phi(x)\right], \\
L^{\prime} & =-\frac{1}{2} \int d^{d} x\left[\pi(x) \vec{x} \cdot \vec{\nabla}_{x} \phi(x)+\vec{x} \cdot \vec{\nabla}_{x} \phi(x) \pi(x)+\frac{d-1}{2} \phi(x) \pi(x)+\frac{d-1}{2} \pi(x) \phi(x)\right] .
\end{aligned}
$$

Note that the latter is the same as the dilatation operator in the CFT. It is easy to see that the massive Hamiltonian $H_{M}=\frac{1}{2} \int d^{d} x\left[\pi(x)^{2}+M^{2} \phi(x)^{2}\right]$ and the IR state $|\Omega\rangle$ is invariant under the transformation by $L$. Their actions on the creation/annihilation operators are given by

$$
\begin{aligned}
e^{-i u L^{\prime}} a_{k} e^{i u L^{\prime}} & =e^{-\frac{d}{2} u} a_{k e^{-u}}, \\
e^{-i u L} a_{k} e^{i u L} & =e^{-\frac{d}{2} u}\left(\cosh (u / 2) a_{k e^{-u}}+\sinh (u / 2) a_{-k e^{-u}}^{\dagger}\right) .
\end{aligned}
$$

From the analysis of [17], we find

$$
\begin{cases}{\left[\cosh \frac{u}{2} a_{k}-\sinh \frac{u}{2} a_{-k}^{\dagger}\right]|\Phi(u)\rangle=0,} & \text { for } k \leq \Lambda e^{u}, \\ {\left[\alpha_{k} \cdot a_{k}+\beta_{k} \cdot a_{-k}^{\dagger}\right]|\Phi(u)\rangle=0,} & \text { for } k \geq \Lambda e^{u} .\end{cases}
$$


By performing the $L$ transformation, we can rewrite these into

$$
\begin{cases}a_{k e^{-u}}|\Psi(u)\rangle=0, & \text { for } k \leq \Lambda e^{u}, \\ {\left[\alpha_{k e^{-u}} a_{k e^{-u}}+\beta_{k e^{-u}} a_{-k e^{-u}}^{\dagger}\right]|\Psi(u)\rangle=0,} & \text { for } k \geq \Lambda e^{u} .\end{cases}
$$

Therefore, in the massless scalar field example, we can explicitly find (when we decompose the state into momentum basis)

$$
\begin{aligned}
|\Phi(u)\rangle & \propto \prod_{k<\Lambda e^{u}} \exp \left(\tanh \frac{u}{2} a_{k}^{\dagger} a_{-k}^{\dagger}\right)|0\rangle \cdot \prod_{k>\Lambda e^{u}}\left|\Omega_{\Lambda}\right\rangle, \\
|\Psi(u)\rangle & \propto \prod_{k<\Lambda}|0\rangle \cdot \prod_{k>\Lambda}\left|\Omega_{\Lambda}\right\rangle,
\end{aligned}
$$

where we recall that $\left|\Omega_{\Lambda}\right\rangle$ is defined in (B.3).

It is important to note that the cMERA state $|\Psi(u)\rangle$ in the CFT is given by the unentangled state $\left|\Omega_{\Lambda}\right\rangle$ only for $k>\Lambda$. However, for $k<\Lambda$ we find that $|\Psi(u)\rangle$ is given by the CFT vacuum $|0\rangle$. This looks at first confusing if we take the IR limit $u=u_{I R} \rightarrow-\infty$ because $\left|\Psi\left(u_{I R}\right)\right\rangle$ should be the unentangled state. However, in the definition of $|\Psi(u)\rangle$ we always perform the non-relativistic scale transformation $L$ under the coarse-graining procedure and thus we only have the disentangled state for the short wave length $k>\Lambda$ for any $u$ (refer to figure 2). Moreover, we should have $|\Psi(u)\rangle=|0\rangle$ for $k\langle\Lambda$ so that $K(u)+L$ becomes identical to the relativistic scale transformation $L^{\prime}$. As we will see in the next subsection, we will not have this issue for the massive scalar example.

On the other hand, if we take the IR limit $u=u_{I R} \rightarrow-\infty$, then we find $\left|\Phi\left(u_{I R}\right)\right\rangle=$ $\left|\Omega_{\Lambda}\right\rangle$ for any $k$. This is because we did not perform any scale transformation to define $|\Phi(u)\rangle$. Notice also these facts are consistent with the fact that $|\Psi(0)\rangle=e^{i u L^{\prime}}|\Psi(u)\rangle$ for $k \leq \Lambda$, where $L^{\prime}$ is the CFT scale transformation.

Finally, note that from the above definition of $L^{\prime}$, it is clear that $L^{\prime}$ annihilates both the vacuum $|0\rangle$ and boundary state $|B\rangle$ :

$$
L^{\prime}|0\rangle=L^{\prime}|B\rangle=0,
$$

where the boundary state (for Dirichlet boundary condition) is given up to the overall normalization constant by:

$$
|B\rangle \propto \prod_{k} \exp \left(-a_{k}^{\dagger} a_{-k}^{\dagger}\right)|0\rangle
$$

\section{B.3 Massive scalar case}

Here we generalize the massless results in the previous subsection into those in the massive scalar case. The scale transformations are given by

$$
\begin{aligned}
e^{-i u L} a_{k} e^{i u L} & =e^{-\frac{d u}{2}}\left[\sqrt{\frac{\epsilon_{k}}{\epsilon_{k e^{-u}}}}\left(a_{k e^{-u}}+a_{-k e^{-u}}^{\dagger}\right)+\sqrt{\frac{\epsilon_{k e^{-u}}}{\epsilon_{k}}}\left(a_{k e^{-u}}-a_{-k e^{-u}}^{\dagger}\right)\right], \\
e^{-i u L^{\prime}} a_{k} e^{i u L^{\prime}} & =e^{-\frac{d u}{2}}\left[e^{-\frac{u}{2}} \sqrt{\frac{\epsilon_{k}}{\epsilon_{k e^{-u}}}}\left(a_{k e^{-u}}+a_{-k e^{-u}}^{\dagger}\right)+e^{\frac{u}{2}} \sqrt{\frac{\epsilon_{k e^{-u}}}{\epsilon_{k}}}\left(a_{k e^{-u}}-a_{-k e^{-u}}^{\dagger}\right)\right] .
\end{aligned}
$$


The state $|\Phi(u)\rangle$ satisfies

$$
\begin{cases}{\left[\left(\sqrt{\epsilon_{\Lambda} / \epsilon_{\Lambda e^{u}}}+\sqrt{\epsilon_{\Lambda e^{u}} / \epsilon_{\Lambda}}\right) a_{k}-\left(\sqrt{\epsilon_{\Lambda} / \epsilon_{\Lambda e^{u}}}-\sqrt{\epsilon_{\Lambda e^{u}} / \epsilon_{\Lambda}}\right) a_{-k}^{\dagger}\right]|\Phi(u)\rangle=0} & \text { for } k \leq \Lambda e^{u}, \\ {\left[\alpha_{k} \cdot a_{k}+\beta_{k} \cdot a_{-k}^{\dagger}\right]|\Phi(u)\rangle=0} & \text { for } k \geq \Lambda e^{u} .\end{cases}
$$

On the other hand, the state $|\Psi(u)\rangle$ is found to be

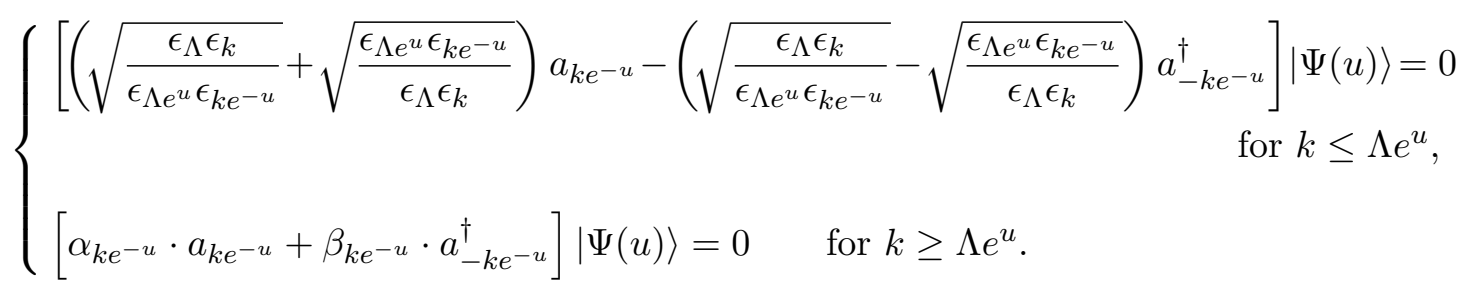

Thus we find for $k \leq \Lambda$

$$
\begin{aligned}
|\Phi(0)\rangle & =|0\rangle, & & \left|\Phi\left(u_{I R}\right)\right\rangle=\left|\Omega_{M_{m}}\right\rangle, \\
|\Psi(0)\rangle & =|0\rangle, & & \left|\Psi\left(u_{I R}\right)\right\rangle=\left|\Omega_{M_{m}}\right\rangle,
\end{aligned}
$$

where we employed the properties in the IR limit $u=u_{I R} \rightarrow-\infty$ :

$$
\Lambda \cdot e^{u_{I R}}=0, \quad k \cdot e^{u_{I R}}=0
$$

Note also that for $k>\Lambda e^{u}$, we simply get $|\Phi(u)\rangle=\left|\Omega_{M_{m}}\right\rangle$ for any $u$. And for $k>\Lambda$ we have $|\Psi(u)\rangle=\left|\Omega_{M_{m}}\right\rangle$ for any $u$. In this way, for massive theories, for any momentum we obtain $|\Psi(u)\rangle=\left|\Omega_{M_{m}}\right\rangle$ as opposed to the massless case.

\section{B.4 Circle compactification}

We can equally analyze the cMERA when the scalar field theory is defined on the spacetime cylinder $\mathbb{R} \times S^{1}$ (for the case of $(1+1) \mathrm{d}$ ). The creation and annihilation operators in the UV theory are denoted by $a_{n / R}^{\dagger}$ and $a_{n / R}$, where $n$ is an arbitrary integer and $R$ is the radius of the cylinder. From the viewpoint of the state $|\Psi(u)\rangle$, at the scale $u$ the radius of the cylinder effectively changes into $R e^{u}$. Therefore the creation and annihilation operators look like $a_{n e^{-u} / R}^{\dagger}$ and $a_{n e^{-u} / R}$. Thus the state $|\Psi(u)\rangle$ is simply given by replacing $k$ with $n / R$ in (B.10). On the other hand, in the description by the state $|\Phi(u)\rangle$, the radius of the cylinder is always $R$ for any $u$. Thus we just need to replace $k$ with $n / R$ in (B.9) and (B.11).

Open Access. This article is distributed under the terms of the Creative Commons Attribution License (CC-BY 4.0), which permits any use, distribution and reproduction in any medium, provided the original author(s) and source are credited. 


\section{References}

[1] G. 't Hooft, Dimensional reduction in quantum gravity, gr-qc/9310026 [INSPIRE].

[2] L. Susskind, The world as a hologram, J. Math. Phys. 36 (1995) 6377 [hep-th/9409089] [INSPIRE].

[3] D. Bigatti and L. Susskind, TASI lectures on the holographic principle, hep-th/0002044 [INSPIRE].

[4] J.M. Maldacena, The Large- $N$ limit of superconformal field theories and supergravity, Int. J. Theor. Phys. 38 (1999) 1113 [Int. J. Theor. Phys. 38 (1999) 1113] [hep-th/9711200] [INSPIRE].

[5] S. Ryu and T. Takayanagi, Holographic derivation of entanglement entropy from AdS/CFT, Phys. Rev. Lett. 96 (2006) 181602 [hep-th/0603001] [INSPIRE].

[6] S. Ryu and T. Takayanagi, Aspects of holographic entanglement entropy, JHEP 08 (2006) 045 [hep-th/0605073] [INSPIRE].

[7] V.E. Hubeny, M. Rangamani and T. Takayanagi, A covariant holographic entanglement entropy proposal, JHEP 07 (2007) 062 [arXiv:0705.0016] [INSPIRE].

[8] T. Nishioka, S. Ryu and T. Takayanagi, Holographic entanglement entropy: an overview, J. Phys. A 42 (2009) 504008 [arXiv:0905.0932] [INSPIRE].

[9] T. Takayanagi, Entanglement entropy from a holographic viewpoint, Class. Quant. Grav. 29 (2012) 153001 [arXiv:1204.2450] [INSPIRE].

[10] M. Van Raamsdonk, Comments on quantum gravity and entanglement, arXiv:0907.2939 [INSPIRE].

[11] M. Van Raamsdonk, Building up spacetime with quantum entanglement, Gen. Rel. Grav. 42 (2010) 2323 [Int. J. Mod. Phys. D 19 (2010) 2429] [arXiv:1005. 3035] [inSPIRE].

[12] G. Vidal, Entanglement renormalization, Phys. Rev. Lett. 99 (2007) 220405 [cond-mat/0512165] [INSPIRE].

[13] G. Vidal, Entanglement renormalization: an introduction, arXiv:0912.1651.

[14] G. Evenbly and G. Vidal, Quantum criticality with the multi-scale entanglement renormalization ansatz, arXiv:1109.5334.

[15] B. Swingle, Entanglement renormalization and holography, Phys. Rev. D 86 (2012) 065007 [arXiv:0905.1317] [INSPIRE].

[16] J. Haegeman, T.J. Osborne, H. Verschelde and F. Verstraete, Entanglement renormalization for quantum fields in real space, Phys. Rev. Lett. 110 (2013) 100402 [arXiv:1102.5524] [INSPIRE].

[17] M. Nozaki, S. Ryu and T. Takayanagi, Holographic geometry of entanglement renormalization in quantum field theories, JHEP 10 (2012) 193 [arXiv:1208.3469] [INSPIRE].

[18] X.-L. Qi, Exact holographic mapping and emergent space-time geometry, arXiv:1309.6282 [INSPIRE].

[19] J. Molina-Vilaplana and P. Sodano, Holographic view on quantum correlations and mutual information between disjoint blocks of a quantum critical system, JHEP 10 (2011) 011 [arXiv:1108.1277] [INSPIRE]. 
[20] J. Molina-Vilaplana, Connecting entanglement renormalization and gauge/gravity dualities, arXiv:1109.5592 [INSPIRE].

[21] J. Molina-Vilaplana, Holographic geometries of one-dimensional gapped quantum systems from tensor network states, JHEP 05 (2013) 024 [arXiv:1210.6759] [INSPIRE].

[22] H. Matsueda, M. Ishihara and Y. Hashizume, Tensor network and a black hole, Phys. Rev. D 87 (2013) 066002 [arXiv:1208.0206] [InSPIRE].

[23] H. Matsueda, Multiscale entanglement renormalization ansatz for Kondo problem, arXiv:1208.2872 [INSPIRE].

[24] B. Swingle, Constructing holographic spacetimes using entanglement renormalization, arXiv:1209.3304 [INSPIRE].

[25] T. Hartman and J. Maldacena, Time evolution of entanglement entropy from black hole interiors, JHEP 05 (2013) 014 [arXiv: 1303.1080] [INSPIRE].

[26] A. Mollabashi, M. Nozaki, S. Ryu and T. Takayanagi, Holographic geometry of cMERA for quantum quenches and finite temperature, JHEP 03 (2014) 098 [arXiv:1311.6095] [INSPIRE].

[27] J. de Boer, M. Kulaxizi and A. Parnachev, Holographic entanglement entropy in Lovelock gravities, JHEP 07 (2011) 109 [arXiv:1101.5781] [INSPIRE].

[28] L.-Y. Hung, R.C. Myers and M. Smolkin, On holographic entanglement entropy and higher curvature gravity, JHEP 04 (2011) 025 [arXiv:1101.5813] [INSPIRE].

[29] X. Dong, Holographic entanglement entropy for general higher derivative gravity, JHEP 01 (2014) 044 [arXiv: 1310.5713] [INSPIRE].

[30] J. Camps, Generalized entropy and higher derivative gravity, JHEP 03 (2014) 070 [arXiv: 1310.6659] [INSPIRE].

[31] C. Holzhey, F. Larsen and F. Wilczek, Geometric and renormalized entropy in conformal field theory, Nucl. Phys. B 424 (1994) 443 [hep-th/9403108] [INSPIRE].

[32] P. Calabrese and J.L. Cardy, Entanglement entropy and quantum field theory, J. Stat. Mech. 0406 (2004) P06002 [hep-th/0405152] [INSPIRE].

[33] J.L. Cardy, Boundary conditions, fusion rules and the Verlinde formula, Nucl. Phys. B 324 (1989) 581 [INSPIRE].

[34] N. Ishibashi, The boundary and crosscap states in conformal field theories, Mod. Phys. Lett. A 4 (1989) 251 [inSPIRE].

[35] L.A. Pando Zayas and N. Quiroz, Left-right entanglement entropy of boundary states, JHEP 01 (2015) 110 [arXiv:1407.7057] [inSPIRE].

[36] A. Kitaev and J. Preskill, Topological entanglement entorpy, Phys. Rev. Lett. 96 (2006) 110404 [hep-th/0510092] [INSPIRE].

[37] M. Levin and X.G. Wen, Detecting topological order in a ground state wave function, Phys. Rev. Lett. 96 (2006) 110405 [cond-mat/0510613].

[38] P. Calabrese and J.L. Cardy, Evolution of entanglement entropy in one-dimensional systems, J. Stat. Mech. 0504 (2005) P04010 [cond-mat/0503393] [INSPIRE].

[39] X.-L. Qi, H. Katsura, and A.W.W. Ludwig, General relationship between the entanglement spectrum and the edge state spectrum of topological quantum states, Phys. Rev. Lett. 108 (2012) 196402 [arXiv:1103.5437]. 
[40] T. Takayanagi and T. Ugajin, Measuring black hole formations by entanglement entropy via coarse-graining, JHEP 11 (2010) 054 [arXiv: 1008.3439] [INSPIRE].

[41] I. Affleck and A.W.W. Ludwig, Universal noninteger 'ground state degeneracy' in critical quantum systems, Phys. Rev. Lett. 67 (1991) 161 [inSPIRE].

[42] M.Z. Hasan and C.L. Kane, Topological insulators, Rev. Mod. Phys. 82 (2010) 3045 [arXiv: 1002.3895] [INSPIRE].

[43] X.L. Qi and S.C. Zhang, Topological insulators and superconductors, Rev. Mod. Phys. 83 (2011) 1057.

[44] A.M. Turner and A. Vishwanath, Beyond band insulators: topology of semi-metals and interacting phases, arXiv: 1301.0330 [INSPIRE].

[45] T. Senthil, Symmetry protected topological phases of quantum matter, Ann. Rev. Condensed Matter Phys. 6 (2015) 299 [arXiv: 1405.4015] [INSPIRE].

[46] F.D.M. Haldane, Continuum dynamics of the $1 D$ Heisenberg antiferromagnet: identification with the $O(3)$ nonlinear sigma model, Phys. Lett. 93A (1983) 464.

[47] F.D.M. Haldane, Nonlinear field theory of large spin Heisenberg antiferromagnets. Semiclassically quantized solitons of the one-dimensional easy Axis Neel state, Phys. Rev. Lett. 50 (1983) 1153 [INSPIRE].

[48] X. Chen, Z.-C. Gu and X.-G. Wen, Classification of gapped symmetric phases in one-dimensional spin systems, Phys. Rev. B 83 (2011) 035107.

[49] F. Pollmann, A.M. Turner, E. Berg and M. Oshikawa, Entanglement spectrum of a topological phase in one dimension, Phys. Rev. B 83 (2010) 064439.

[50] N. Schuch, D. Perez-Garcia and I. Cirac, Classifying quantum phases using matrix product states and projected entangled pair states, Phys. Rev. B 84 (2011) 165139.

[51] F. Pollmann, E. Berg, A.M. Turner and M. Oshikawa, Entanglement spectrum of a topological phase in one dimension, Phys. Rev. B 81 (2010) 064439 [arXiv:0910.1811].

[52] T. Faulkner, A. Lewkowycz and J. Maldacena, Quantum corrections to holographic entanglement entropy, JHEP 11 (2013) 074 [arXiv: 1307.2892] [INSPIRE].

[53] T. Takayanagi, Holographic dual of BCFT, Phys. Rev. Lett. 107 (2011) 101602 [arXiv: 1105.5165] [INSPIRE].

[54] M. Fujita, T. Takayanagi and E. Tonni, Aspects of AdS/BCFT, JHEP 11 (2011) 043 [arXiv:1108.5152] [INSPIRE].

[55] M. Fujita, W. Li, S. Ryu and T. Takayanagi, Fractional quantum Hall effect via holography: Chern-Simons, edge states and hierarchy, JHEP 06 (2009) 066 [arXiv:0901.0924] [INSPIRE].

[56] J. I. Cirac and F. Verstraete, Renormalization and tensor product states in spin chains and lattices, J. Phys. A 42 (2009) 4004 [arXiv:0910.1130].

[57] J.L. Cardy, Operator content of two-dimensional conformally invariant theories, Nucl. Phys. B 270 (1986) 186 [INSPIRE].

[58] I. Heemskerk and J. Polchinski, Holographic and wilsonian renormalization groups, JHEP 06 (2011) 031 [arXiv: 1010.1264] [InSPIRE].

[59] T. Faulkner, H. Liu and M. Rangamani, Integrating out geometry: holographic wilsonian RG and the membrane paradigm, JHEP 08 (2011) 051 [arXiv: 1010.4036] [INSPIRE]. 
[60] S. Ryu and Y. Hatsugai, Entanglement entropy and the Berry phase in the solid state, Phys. Rev. B 73 (2006) 245115 [cond-mat/0601237].

[61] A.P. Schnyder, S. Ryu, A. Furusaki and A.W.W. Ludwig, Classification of topological insulators and superconductors in three spatial dimensions, Phys. Rev. B 78 (2008) 195125 [arXiv: 0803.2786].

[62] S. Ryu, A. P. Schnyder, A. Furusaki, A. W. W. Ludwig, Topological insulators and superconductors: ten-fold way and dimensional hierarchy, New J. Phys. 12 (2010) 065010 [arXiv: 0912.2157].

[63] A. Kitaev, Periodic table for topological insulators and superconductors, AIP Conf. Proc. 1134 (2009) 22 [arXiv:0901.2686]. 